\title{
Visual Detection of Change Points and Trends Using Animated Bubble Charts
}

\author{
Sackmone Sirisack and Anders Grimvall \\ Linköping University, \\ Sweden
}

\section{Introduction}

The rapid growth of automatic data collection systems has increased the need for algorithms that can efficiently reveal important features of large or complex datasets. For example, it is often of great interest to examine the occurrence of abrupt changes in long bi- or multivariate time series of data. Several numerical algorithms and statistical tests have been developed to detect abrupt shifts in the mean or other parameters of uni- or multivariate distributions (Caussinus \& Mestre, 2004; Hawkins, 1977, 2001; Srivastava \& Worsley, 1986; Stephens, 1994). However, there is also a need for visualization techniques that can help the user identify any type of abrupt changes or trends in the collected data. More generally, techniques are needed that can simultaneously highlight important features of the data and filter out irrelevant information (Bederson \& Boltman, 1999; Bundesen, 1990; Cleveland \& McGill, 1984; Healey, 2000; Ware, 2004). In this chapter, we present flexible and user-friendly animations of bubble charts in which subsets of the collected data are sequentially highlighted on a static background representing all data points.

The basic ideas of interactive visualization of quantitative data were presented before computer technologies were sufficiently developed to enable widespread use of such methods. In 1978, Newton introduced a form of linked brushing that allowed the user to select a subset of observations in one display and simultaneously highlight the same subset in another display. About a decade later, several ground-breaking articles were published. Asimov (1985) introduced the concept of helicopter tours for viewing highdimensional datasets via a structured progression of 2D projections, and Becker and coworkers $(1987 \mathrm{a}, \mathrm{b})$ provided a systematic framework for brushing, linking, and other forms of interactive statistical graphics. Moreover, Unwin and colleagues (1988) demonstrated how zooming, rescaling, and overlaying can facilitate visual analysis of multivariate time series data.

More recently, improvements in computing power, display resolution, and numerical algorithms have brought interactive visualization of quantitative data to higher levels and stimulated the development of new applications. The software XGobi and its descendant GGobi set a new standard for interactive modification of linked plotting windows, and an application programming interface made such methods available to the rapidly growing group of R users (Cook \& Swayne, 2007; Swayne et al., 2003; the GGobi website, 2011). Zooming and rescaling were established as standard tools in software packages for time 
series analysis, and visual specification of queries was introduced to facilitate the search for interesting features of time series data (Hochheiser et al., 2003).

Motion charts, or animated bubble charts, represent another breakthrough in data visualization (the Gapminder website, 2011). The basic display is a 2D bubble chart showing observed pairs of two variables $x$ and $y$ that have been recorded annually for a set of objects. By highlighting the positions of the bubbles year by year, changes over time can be visualized. Additional information about the investigated objects can be entered into the graphs by colour-coding the bubbles and letting their size vary with some covariate. A Google gadget (the Google website, 2011) has made motion charts available to any user with a good Internet connection.

The use of animated population pyramids in official statistics (the Australian Bureau of Statistics, 2011) illustrates that almost any static graph in statistics can be animated to visualize changes over time. However, some authors have emphasized that animations are not always superior to static presentations such as a small multiples display (Robertson et al., 2008). Visualization of temporal changes in the size and shape of 2D point clouds represents yet another approach that is particularly suitable for exploring large datasets (Landesberger et al., 2009).

Here, we present a flexible two-stage method for making animated bubble charts in Excel ${ }^{\circledR}$. In the first stage, a macro written in VBA (Visual Basic for Applications) is utilized to identify data tables in a given worksheet and help the user select and organize the inputs to the animation. This macro also creates a suitable bubble-chart template. Thereafter, a collection of other VBA macros is employed to produce the animation.

The methods and software solutions we propose are designed to handle fairly large datasets with multiple groups of objects and multiple observations per time stamp and group. Furthermore, it can be noted that the order in which different subsets of data are highlighted can be determined by an arbitrary numerical or string variable. In general, bubble charts are used to visualize relationships between interval variables. However, relationships involving categorical or ordinal variables can also be visualized. In such cases, adding a small amount of noise (jitter) to the original data might be helpful, because it will improve the separation of the data points so that each point is made visible. In addition, the visualization can be extended to high-dimensional time series data by using a macro that first performs principal components analysis and then creates $2 \mathrm{D}$ animated score charts.

After a brief summary of the general principles of animating bubble charts, and some remarks regarding design issues, we use time series of daily to monthly environmental data to illustrate the power of visual tools to bring out important characteristics of the collected data. Most of our analyses are focused on the occurrence of sudden shifts in the mean or dispersion, and whether or not such shifts can be found in all investigated groups of data. However, the tools presented here are also used to examine temporal trends across seasons and changes along gradients. Moreover, we use a set of multivariate chemical data on olive oils to illustrate how animated score charts can highlight differences between geographical regions.

After presenting a set of useful displays and animation options, we resume our discussion of factors that influence the visual impression of static and animated charts, and we also consider how to achieve a good balance between the information content of a display and perceptual capacity limits. In addition, we address some technical aspects of using spreadsheets with tens of thousands of observations. 


\section{General principles of animating bubble charts}

In Excel ${ }^{\circledR}$ and other spreadsheet programs, graphs added to a worksheet can be updated automatically and almost instantaneously when the content of the worksheet is altered. This enables animations driven by a macro that achieves step-by-step changes in the content of a range of worksheet cells. The speed of an animation can be controlled by making calls to a special function that puts the macro to sleep and wakes it up after a specified amount of time.

Because visual inspection is particularly suitable for detecting motion against a static background, we developed animations in which all data are used to construct a static background, and different subsets of data are sequentially highlighted. In a 2D bubble chart, this type of displays can be constructed by using open markers for the static background and filled markers for the highlighted data. This is illustrated in Figure 1, which shows how the interdependence between reported $\mathrm{pH}$ and alkalinity levels in the Baltic Proper has changed over time. In particular, it can be noted that the reported interdependence changed dramatically from 1989-1993 to 1994-1998, most probably due to changes in laboratory practices.

\section{Some design issues}

A user-friendly implementation of animated bubble charts requires a good balance between flexibility and standardization. The selection of data and the design of the bubble charts should be flexible, whereas efficient updating of spreadsheets and graphs is greatly facilitated if the data tables have a standardized design. This favours two-stage procedures in which a set of user forms first help the user organize the data in a standardized manner and create a suitable graph template; thereafter, the animation can be run and controlled with buttons and scroll bars.

We created a VBA macro that initially determines the position and size of the data tables that are to be visualized, and then utilizes list boxes to select up to five variables for an animated bubble chart. The first variable, which is required and may represent a time stamp, is used to control the highlighting of different subsets of data. Variables two and three, which are also required, represent the $x$ and $y$ variables in a bubble chart. Variable four, which is optional, can be used to partition the set of bubbles into different groups. Finally, another optional variable can be used to size code the bubbles.

The macro that prepares for the animation can also allow the user to select a suitable step length (time step) for the animation and a desired range of animation records (time span). Furthermore, the preparations include automatic scaling of the $x$ - and $y$-axes of the bubble chart and selection of marker types. The applicability of animated bubble charts can be further increased by performing an optional standardization of the $x$ and $y$ variables to mean zero and variance one, and by calculating the first two principal components of a userdefined set of variables. In the latter case, high-dimensional data can be scrutinized by creating animated 2D score charts.

\section{Different types of displays}

\subsection{Standard bubble charts with groups}

The simplest form of bubble charts has a single group of highlighted cases (see Fig. 1). This type of display can easily be generalized to displays in which two or more groups are 
assigned different coloured markers. Theoretically, the red-green-blue (RGB) system enables colour coding of up to $2^{24}$ groups. However, static bubble charts with more than eight colours are difficult to perceive (Gilmore et al., 1989), and animated charts are best perceived if no more than four groups of cases are simultaneously highlighted in the same display.
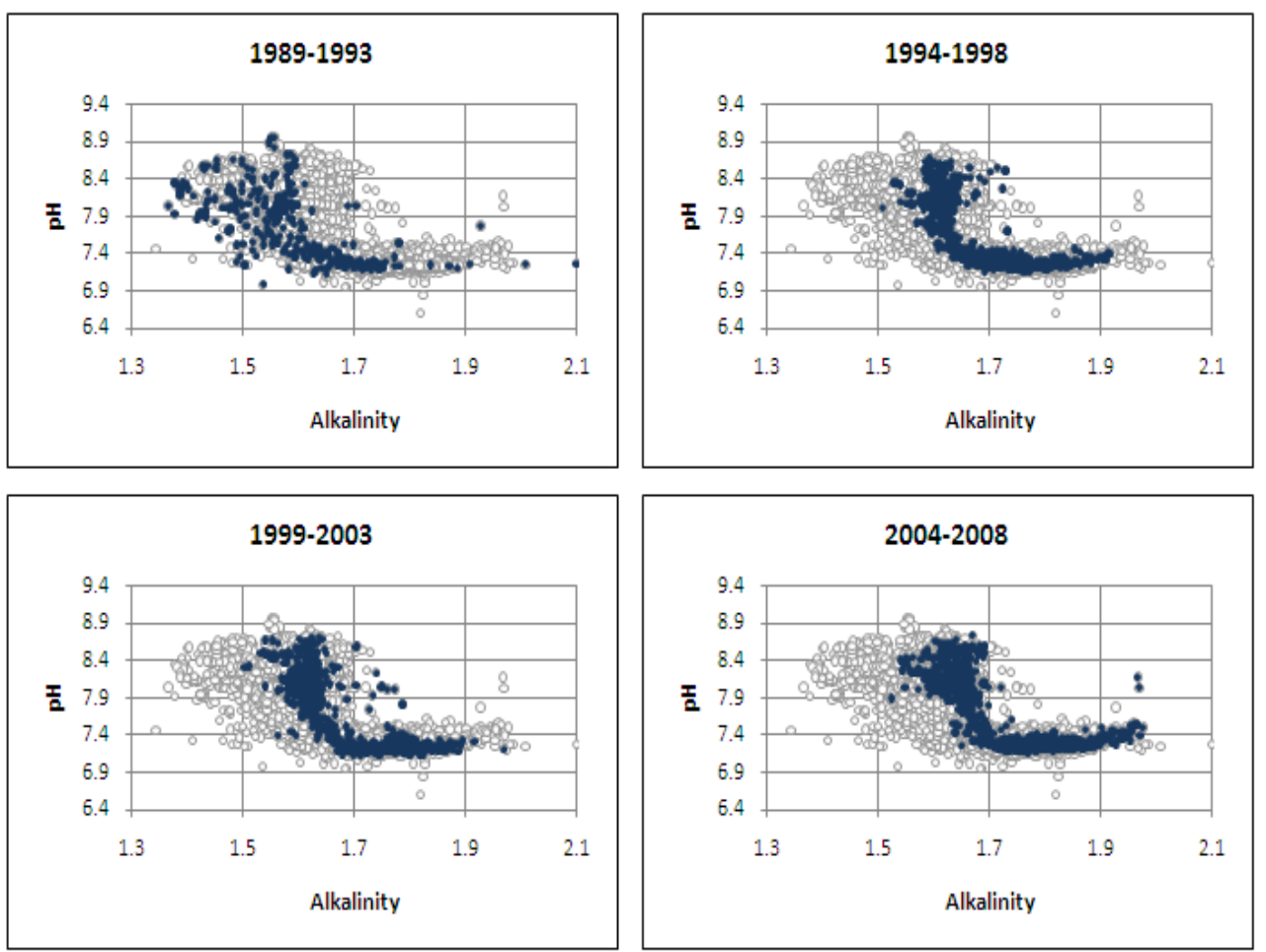

Fig. 1. Four consecutive frames from an animation of $\mathrm{pH}$ against alkalinity of seawater samples from the Eastern Gotland Basin in the Baltic Proper (sampling site BY15). Data source: the Swedish Meteorological and Hydrological Institute (SMHI).

Figure 2 shows how the interdependence between $\mathrm{pH}$ and salinity of seawater samples varied over time and between laboratories. In particular, it can be seen that in 1989-1993 the variability of $\mathrm{pH}$ for a given salinity was unusually large for one of the laboratories involved, which indicates data quality problems. Moreover, there are single outliers in the data that were collected more recently. Further studies are needed to determine whether these outliers represent flawed data or unusual water samples. It cannot be excluded that mixing of seawater due to strong winds can cause rather abrupt changes in $\mathrm{pH}$.

We have already emphasized that multicoloured bubble charts should be used with caution. This advice is further motivated by Figure 3, in which the upper frames with group-specific coloured markers contain more information than the lower frames with black markers only. Nevertheless, the lower frames show more clearly that there was a level shift in the total volume of phytoplankton between the two time periods, although 
the content of chlorophyll-a changed very little. It should also be kept in mind that if different colours are used in the same panel, they may interfere with each other. Spatial patterns in strong colours may conceal patterns in light colours, if the background is white.
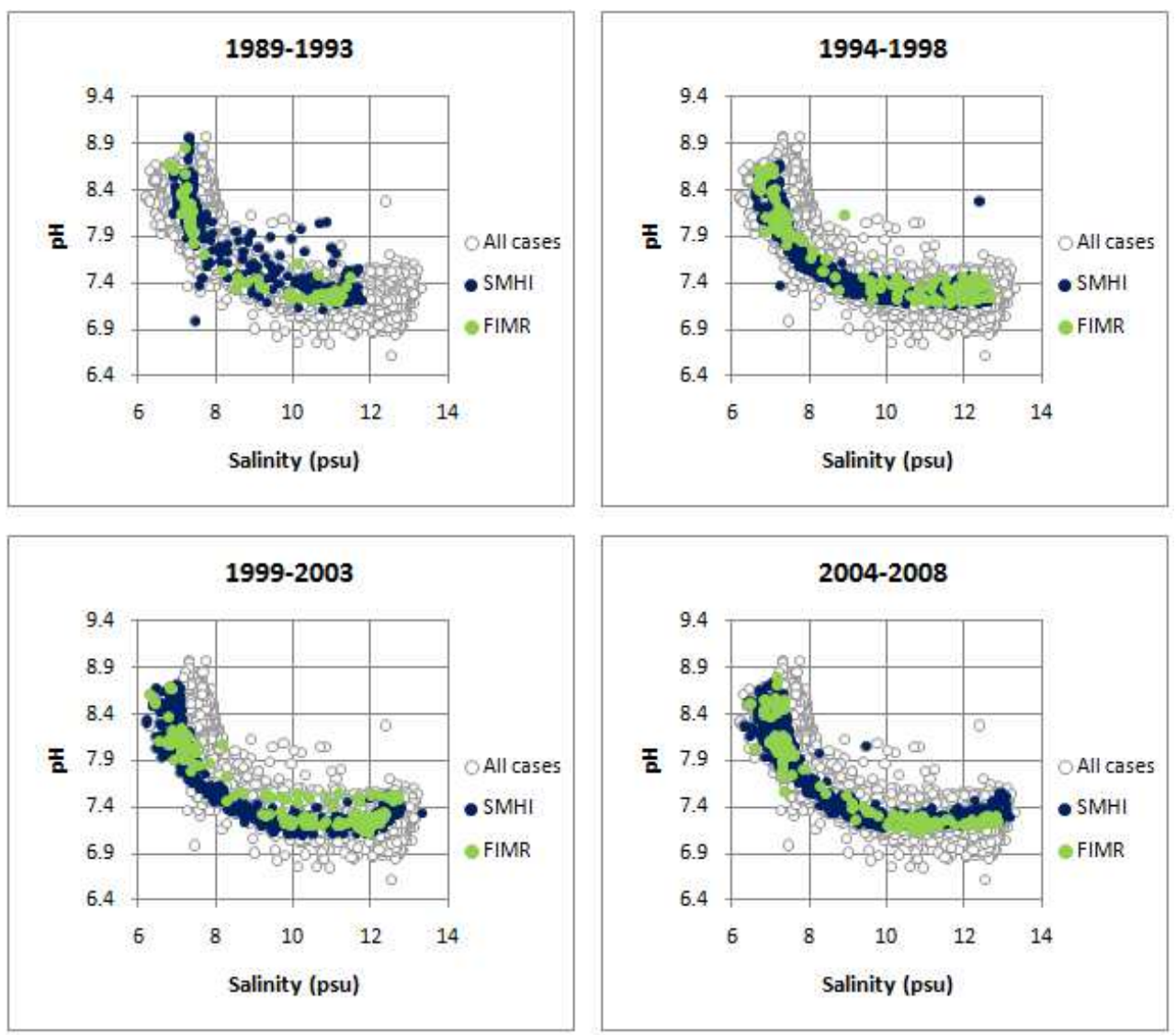

Fig. 2. Four consecutive frames from an animation of salinity and $\mathrm{pH}$ data for seawater samples collected in the Eastern Gotland Basin in the Baltic Proper (sampling site BY15) and analysed by the Swedish Meteorological and Hydrological Institute (SMHI) and the Finnish Institute of Marine Research (FIMR).

Size-coding of bubble chart markers is another tool that should be employed with great caution, unless the user actually wants to suppress some data points or the dataset is so small that the markers can be inspected one by one. Furthermore, it is worth noticing that the (average) size of the markers has a strong impact on the perception of a pattern formed by a set of markers. Markers that are too small tend to blur the contours of a cloud of points, and large markers can make it difficult to comprehend the number of points in different subsets of data. 

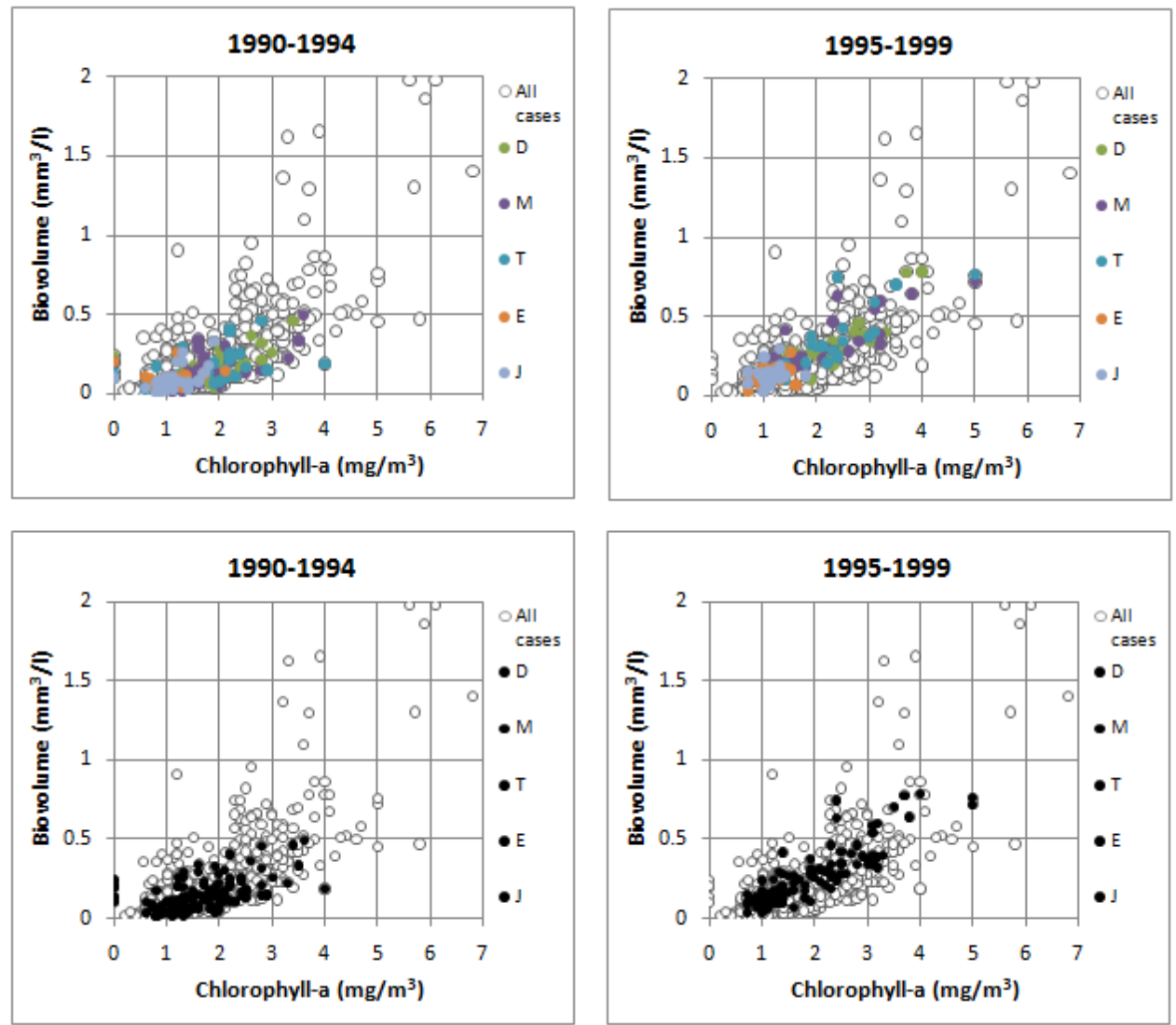

Fig. 3. Bubble charts of phytoplankton data from three sites in Lake Vänern (D, Dagskärsgrund N; M, Megrundet N; T, Tärnan SSO) and two sites in Lake Vättern (E, Edeskvarnaån NV; J, Jungfrun NV) in Sweden. The coloured markers in the upper panels have been changed to black markers in the lower panels. Data source: the Swedish University of Agricultural Sciences (SLU).

\subsection{Jittered bubble charts}

A jittered plot adds some random noise to the $\mathrm{x}$ or the $\mathrm{y}$ coordinate, or both. Such plots are particularly useful for categorical and ordinal data, because they can give a realistic visual impression of the number of cases in different parts of the chart. In environmental monitoring, jittered plots are particularly useful when the $x$ coordinate represents a class variable such as month or season, or the y coordinate represents a count variable such as the number of species found in the analysed sample.

Figure 4 illustrates a suspected artificial level shift in temperature data from the Czech Republic. The time series plot indicates that the temperature difference between the two investigated meteorological stations increased in 1998. By using a jittered plot to visualize the differences by month, it can be seen that the level shift was present during all seasons and was particularly pronounced during the warmer months. 

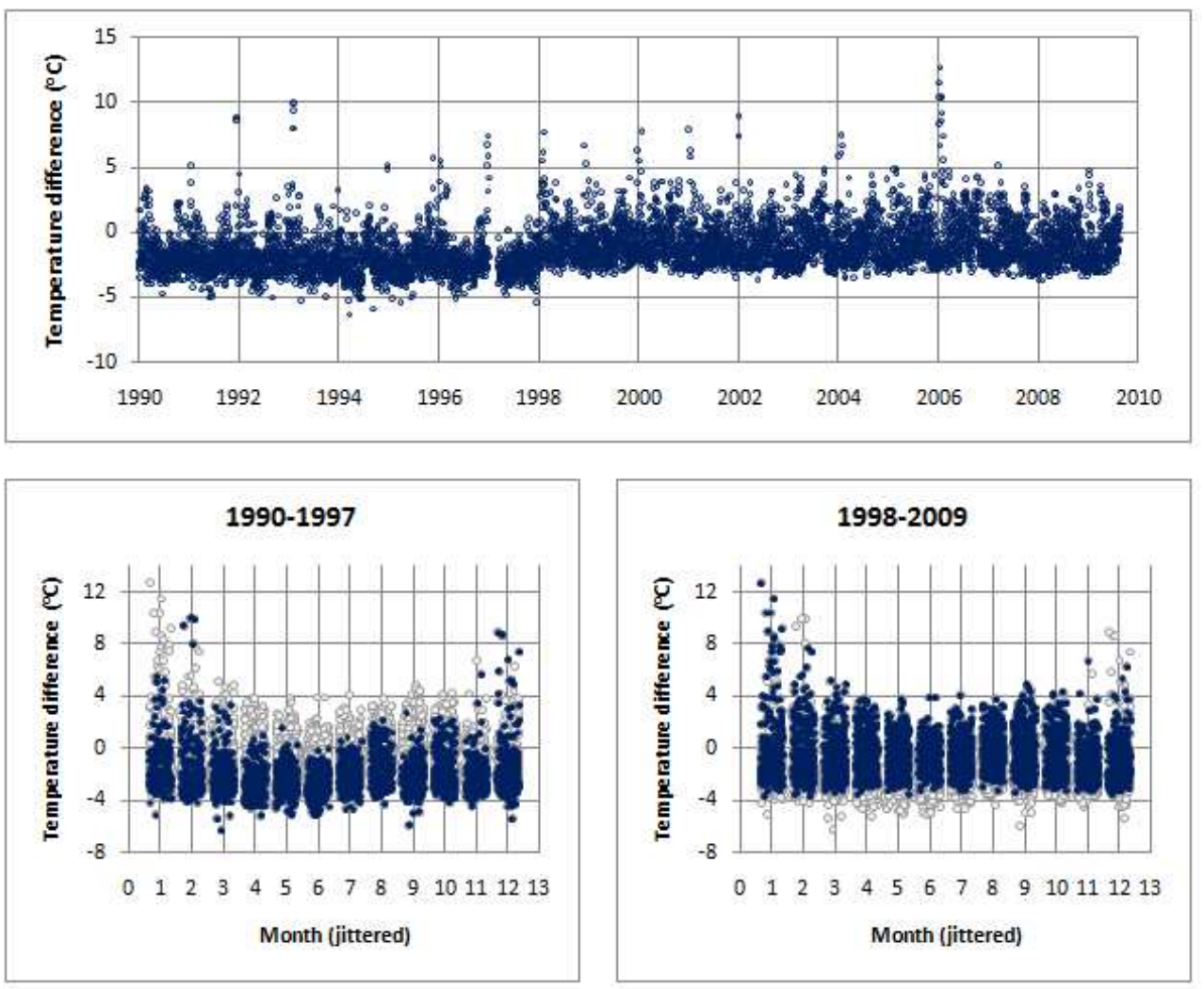

Fig. 4. Ordinary time series plot and jittered bubble charts of the difference in daily mean temperatures between the meteorological stations Protivanov and Jevičko in the Czech Republic. A small amount of noise has been added to the month number. Data source: the Czech Hydrometeorological Institute, Brno.

\subsection{Bubble charts with trend lines}

When there is pronounced seasonal variation in the collected data, it may be of interest to animate changes in trend slopes by month. This can be achieved by using the month as animation variable and one of the built-in trend line options in Excel®. Figure 5 shows longterm temperature trends in central England, and the four panels draw attention to the fact that the trend slope gradually decreases from March to June. In principle, this pattern could have been revealed by producing a series of static plots. However, this process can be automated by using software for animation. In addition, animation can help to identify between which months of the year that the major changes in trend slopes occur. Such differences in slopes between adjacent months can be further accentuated by standardizing the data so that differences in monthly means are eliminated. 

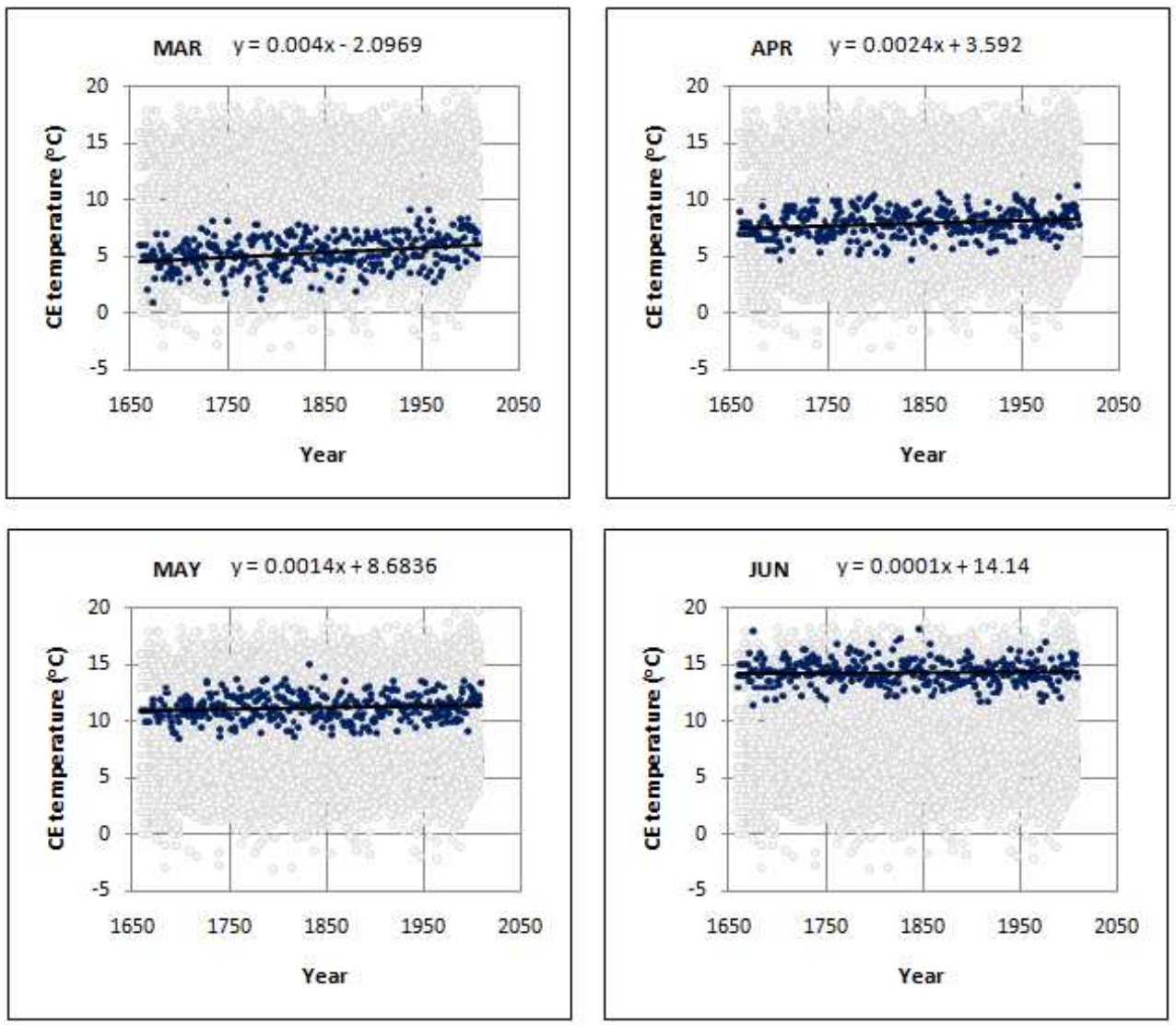

Fig. 5. Four consecutive frames from an animation of trends by month for the Central England Temperature series compiled by the Hadley Centre, UK.

\subsection{Gradient charts}

In many environmental monitoring programmes, the sampling sites have a natural order. For example, samples from the marine environment are often taken along salinity or depth gradients, air pollutants are measured at different distances from a point source, and river water quality can be measured at different runoff levels. This calls for techniques that can efficiently visualize how relationships between two or more variables change along a gradient.

Figure 6 illustrates in two different manners how the relationship between the concentrations of phosphorus and suspended matter in a small stream varies with the runoff level. It is obvious that, compared to a static chart in which colour- and shapecoded markers are used to indicate runoff levels, an animated display has two advantages. First, there is no perceptual interference between the different subsets of data. Second, the analyst can inspect one highlighted subset while the previous subset is still fresh in memory. 

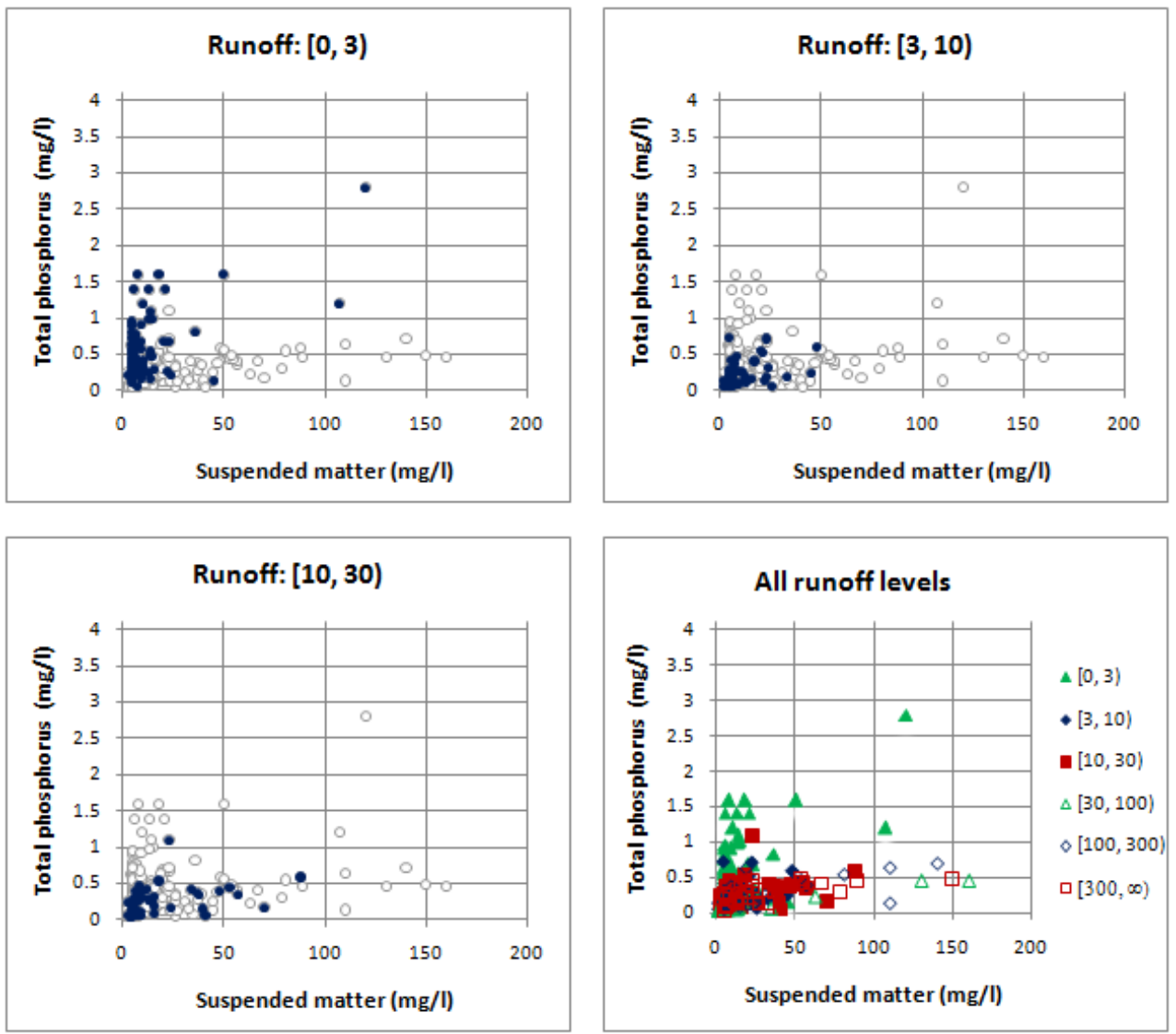

Fig. 6. Relationship between the concentrations of phosphorus and suspended matter in stream water from an agriculture-dominated catchment in southern Sweden. Data source: the Swedish University of Agricultural Sciences (SLU), catchment code N33.

\subsection{Score charts for a pair of principal components}

When the collected data are multivariate and the coordinates are strongly correlated, important information can be obtained from score charts in the coordinate system determined by the first two principal components. An animation can refine such information by highlighting data points by time or group. As in the gradient plots in the previous section, the advantage of an animated display is that there is no perceptual interference between the different subsets of data.

Figure 7 shows an animation of regional differences in the chemical composition of olive oil from different regions in Italy. The score charts draw attention to the fact that some groups of objects are more heterogeneous than others. By ordering the regions from south to north, or according to some characteristic of the areas, this type of animations can also highlight various gradients in the chemical composition. 

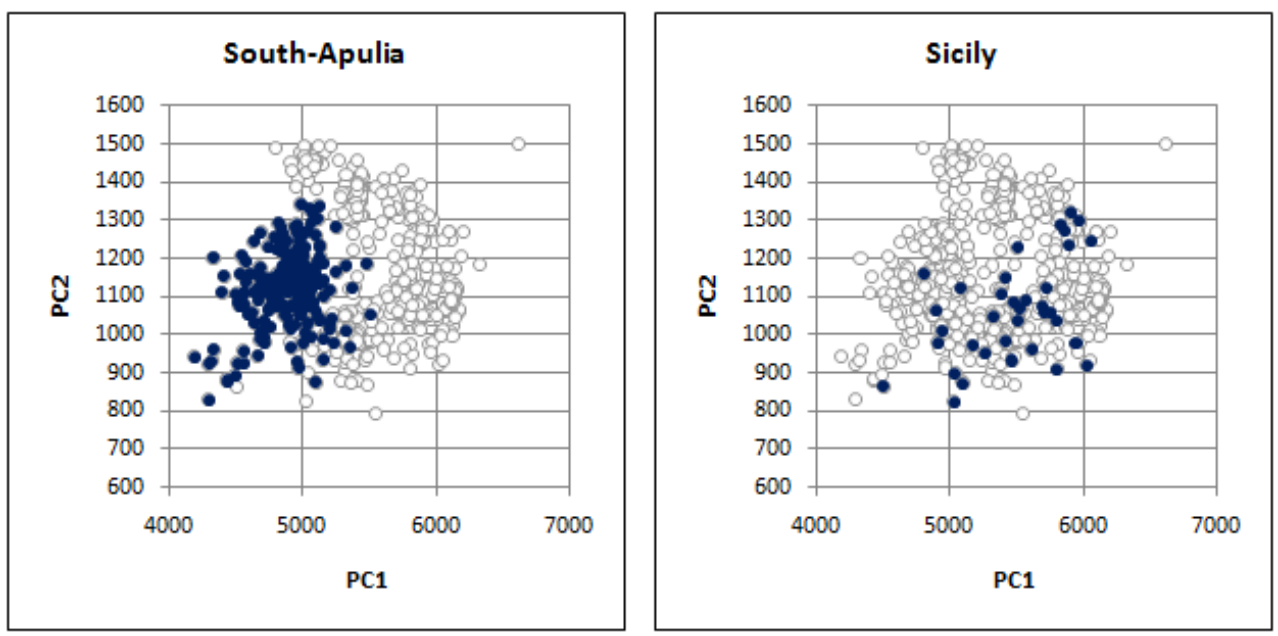

Fig. 7. Two frames from an animation of score charts derived from a dataset containing information about the content of eight different fatty acids in olive oil from nine different regions in Italy. Raw data were obtained from the Ggobi Website.

\section{Computational aspects}

The technical performance of Excel-based animations is markedly influenced by the technique that is used to update the content of the worksheet cells. In particular, the computational time can be reduced considerably, if large arrays are updated by a single command rather than by creating a loop in which individual cells are updated one by one. The performance can also be improved by turning off the automatic screen updating and the automatic calculation of worksheets during parts of the execution of the animation macro.

The design of the markers in the bubble chart is yet another factor that strongly influences the computational time. It takes longer to update large bubbles than small markers, and more elaborate bubbles that resemble $3 \mathrm{D}$ balls can greatly retard the animation.

Test runs using a dataset comprising 10,000 cases showed that a chart with 400 highlighted bubbles could be updated in less than two seconds on a standard PC. If the dataset is substantially larger, it may be preferable to base the animation on a (random) sample of the original data.

\section{Discussion}

When multiple views or complex graphical coding of multivariate data are used to bring loads of information into a single display, there is a considerable risk that the data representation will be visually impenetrable. Displays with multiple views can suffer from visual fragmentation, and perceptual interference can occur between different graphical codes in the same display (Healey, 2000; Bartram, 2001). The animated bubble charts presented in this article represent an attempt to simultaneously reduce visual fragmentation and perceptual interference. 
The static background composed of open markers showing the distribution of the entire dataset enables rapid assessment of the distribution of a highlighted subset of data points. Moreover, the animation facilitates detection of change, because the analyst can inspect the shape and size of a highlighted point cloud while the previous point cloud is still fresh in memory.

Using filled markers of standardized shape makes it easier to discern the colour coding. Further, perception of a scatter plot can be strongly affected by the size of the markers, and hence it is worth noting that the built-in scaling feature in Excel can be used to reduce or increase the size of the bubbles in the charts. However, as emphasized in the introduction, only a few different colours and bubble sizes can be readily distinguished by visual inspection, and there may be perceptual interference between colour and size coding (Healey, 2000; Bartram, 2001). In addition, it should be mentioned that static visualizations, such as a small multiples display, are still viable alternatives to animated graphs (Robertson et al., 2008).

Much of the work presented here was inspired by Rosling and co-workers (Gapminder, 2011), who demonstrated that the animated bubble chart is a powerful tool for visualizing temporal trends in official statistics and other data collected annually for a set of objects. When one variable is plotted against another, and a video is created to simultaneously display changes over the period of data collection, the motion of the bubbles can draw attention to subsets of objects that move simultaneously in the same direction. Similarly, the motion makes it easier to identify deviating objects that move in a completely different direction.

Our work here has demonstrated that animated bubble charts are also very useful for inspecting temporal changes in the shape and size of 2D point clouds. For example, such animations can efficiently reveal changes in the presence of outliers or in the conditional mean and variance of one variable given another. Moreover, detection of change across time or groups can be greatly facilitated if open bubbles representing the entire dataset are allowed to form a static background, while selected subsets of data points are sequentially highlighted at a rate determined by the user.

Also, it should be noted that animated bubble charts can be useful, even if the order of the highlighted subsets lacks meaning. Without writing any computer code, a large number of simple bubble charts can be created and inspected at a pace determined by the analyst. Our animated 2D score charts represent yet another example of a time-saving procedure that can create a good overview of a complex dataset.

This article has focused on construction of animated bubble charts in a spreadsheet program where charts that are added are automatically updated when the contents of some worksheet cells are updated. Other software or programming environments can provide other solutions to animation problems. In $\mathrm{R}$, for instance, a sequence of frames representing different time stamps are combined into a video prior to the animation, whereas the Google gadget Motion Chart provides several means of interaction. The main technical advantages offered by the Excel-based animations presented here are flexibility and the capacity to handle fairly large datasets. Test runs showed that, compared to Google Motion Chart, our tools can handle larger datasets. Furthermore, they are very flexible in three respects: (i) an arbitrary numerical or string variable can be used to determine the order in which different subsets of data are highlighted; (ii) any Excel tool can be used to modify the design of the bubble chart prior to the animation; (iii) multidimensional data can be scrutinized by first performing a principal components 
analysis and then animating a score chart in which the observations are plotted in a coordinate system determined by the first two eigenvectors.

\section{Conclusions}

Our study demonstrated that animated bubble charts can facilitate detection of change points and trends. More specifically, we emphasized that such charts have the following advantages:

i. the analyst can inspect the shape and size of a highlighted point cloud while the previous point cloud is still fresh in memory;

ii. bubble charts in which the entire dataset is allowed to form a static background put the high-lighted subset into a wider perspective;

iii. animations are time-saving procedures that can readily create a good overview of complex datasets.

Furthermore, we showed that our Excel-based software solutions are very flexible in three respects:

i. an arbitrary numerical or string variable can be used to determine the order in which different subsets of data are highlighted;

ii. any Excel tool can be used to modify the design of the bubble chart prior to the animation;

iii. multidimensional data can be scrutinized by first performing a principal components analysis and then animating a score chart in which the observations are plotted in a coordinate system determined by the first two eigenvectors.

In summary, our results demonstrate that animation can simultaneously reduce visual fragmentation and perceptual interference.

\section{Acknowledgements}

The authors are grateful to the Swedish International Development Cooperation Agency (SIDA) and its Department for Research Cooperation (SAREC), and the Faculty of Science (FOS) at the National University of Laos (NUOL) for providing financial support and facilities for this research. We are also grateful to the Swedish University of Agricultural Sciences, the Swedish Meteorological and Hydrological Institute, and the Czech Hydrometeorological Institute for supplying environmental data.

\section{References}

Asimov D. The grand tour: A tool for viewing multidimensional data. SIAM. Journal of Science and Statistical Computing 1985; 6:128-143.

Bartram LR. Enhancing information visualization with motion. PhD Thesis, School of Computer Science, Simon Fraser University, 2001.

Becker RA, Cleveland WS. Brushing scatterplots. Technometrics 1987; 29:127-142.

Becker RA, Cleveland WS, Wilks AR. Dynamic graphics for data analysis. Statistical Science 1987; 2:355-396. 
Bederson B, Boltman A. Does animation help users build mental maps of spatial information? Proceedings of the 1999 IEEE Symposium on Information Visualization (InfoVis 1999), San Francisco, CA, Oct 1999.

Bundesen C. A theory of visual attention. Phsychological Review 1990; 97:523 - 547.

Caussinus H, Mestre O. Detection and correction of artificial shifts in climate series. Applied Statistics 2004; 53:405-425.

Cleveland WS, McGill R. Graphical perception: Theory, experimentation, and application to the development of graphical methods. Journal of the American Statistical Association 1984; 79:531-554.

Cook D, Swayne, DF. 2007. Interactive and Dynamic Graphics for Data Analysis - with R and Ggobi. New York: Springer Verlag.

Gilmore W, Gertman D, Blackman H. User-Computer Interfaces in Process Control: A Human Factors Engineering Handbook. Academic Press: San Diego, 1989.

Hawkins DM. Testing a sequence of observations for a shift in location. Journal of the American Statistical Association 1977; 72:180-186.

Hawkins DM. Fitting multiple change-point models to data. Computational Statistics and Data Analysis 2001; 37:323-341.

Healey CG. Building a perceptual visualisation architecture. Behaviour and Information Technology 2000, 19:349-366.

Hochheiser H, Baehrecke EH, Mount SM, Shneiderman B. Dynamic querying for pattern identification in microarray and genomic data. Proceedings of the IEEE Multimedia Conference and Expo, July 2003, Baltimore, MD, 2003.

Landesberger TV, Bremm S, Rezaei P, Schreck T. Visual analytics of time dependent 2D point clouds. Computer Graphics International 2009; pp 97-101.

Newton C. Graphics: From Alpha to Omega in Data Analysis. In Graphical Representation of Multivariate Data. Academic Press: New York, 1978; pp 59-92.

Robertson G, Fernandez R, Fisher D, Lee B Stasko J. Effectiveness of animation in trend visualization. Visualization and Computer Graphics 2008; 14:1325-1332.

Srivastava MS, Worsley KJ. Likelihood ratio tests for a change in the multivariate normal mean. American Statistical Association 1986; 81:199-204.

Stephens DA. Bayesian retrospective multiple-changepoint identification. Journal of the Royal Statistical Society: Series C 1994; 43:159-178.

Swayne DF, Lang DT, Buja A, Cook D. GGobi: evolving from XGobi into an extensible framework for interactive data visualization. Computational Statistics and Data Analysis 2003; 43:423-444.

The GGobi website. http://www.ggobi.org/ [12 July 2011]

The Gapminder website. http:/ / www.gapminder.org/ [12 July 2011]

The Google website.

https:// docs.google.com/templates?type=spreadsheets\&q=motion+chart\&sort=ho ttest\&view=public [12 July 2011]

The Australian Bureau of Statistics website. http:/ / www.abs.gov.au/websitedbs/d3310114.nsf/home/Population\%20Pyramid $\% 20-\% 20$ Australia [12 July 2011] 
Unwin AR, Wills G. Eyeballing time series. Proceedings of the 1988 ASA Statistical Computing Section 1988; pp 263-268.

Ware C. Information Visualization: Perception for Design (2nd edn). Morgan Kaufmann Publishers, 2004. 


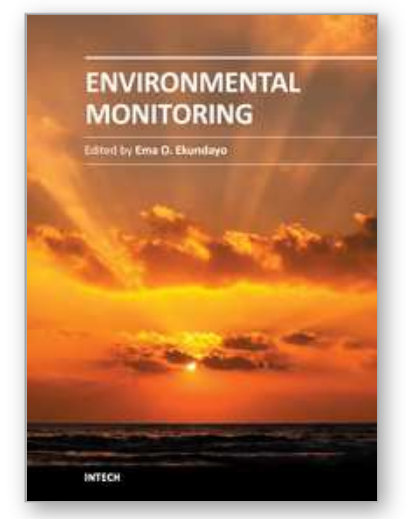

\author{
Environmental Monitoring \\ Edited by Dr Ema Ekundayo
}

ISBN 978-953-307-724-6

Hard cover, 528 pages

Publisher InTech

Published online 04, November, 2011

Published in print edition November, 2011

"Environmental Monitoring" is a book designed by InTech - Open Access Publisher in collaboration with scientists and researchers from all over the world. The book is designed to present recent research advances and developments in the field of environmental monitoring to a global audience of scientists, researchers, environmental educators, administrators, managers, technicians, students, environmental enthusiasts and the general public. The book consists of a series of sections and chapters addressing topics like the monitoring of heavy metal contaminants in varied environments, biolgical monitoring/ecotoxicological studies; and the use of wireless sensor networks/Geosensor webs in environmental monitoring.

\title{
How to reference
}

In order to correctly reference this scholarly work, feel free to copy and paste the following:

Sackmone Sirisack and Anders Grimvall (2011). Visual Detection of Change Points and Trends Using Animated Bubble Charts, Environmental Monitoring, Dr Ema Ekundayo (Ed.), ISBN: 978-953-307-724-6, InTech, Available from: http://www.intechopen.com/books/environmental-monitoring/visual-detection-ofchange-points-and-trends-using-animated-bubble-charts

\section{INTECH}

open science | open minds

\section{InTech Europe}

University Campus STeP Ri

Slavka Krautzeka 83/A

51000 Rijeka, Croatia

Phone: +385 (51) 770447

Fax: +385 (51) 686166

www.intechopen.com

\section{InTech China}

Unit 405, Office Block, Hotel Equatorial Shanghai

No.65, Yan An Road (West), Shanghai, 200040, China

中国上海市延安西路65号上海国际贵都大饭店办公楼405单元

Phone: +86-21-62489820

Fax: +86-21-62489821 
(C) 2011 The Author(s). Licensee IntechOpen. This is an open access article distributed under the terms of the Creative Commons Attribution 3.0 License, which permits unrestricted use, distribution, and reproduction in any medium, provided the original work is properly cited. 